\title{
Optimal Portfolio Choice under Hidden Regime Switching Model
}

\author{
Zhiying Chen Xuanhua Peng Yongkui Li \\ School of Economics, Southwest University of Political Science \& Law, Chongqing \\ 401120, China
}

\begin{abstract}
We investigate a portfolio optimization problem in a continuous-time Markov-modulated financial market. The unobservable mean return of a risky asset follows a continuous-time, two-state Markov chain whose states are interpreted as different states of market. Using results from filter theory, we reduce this problem to one with complete observation. We solve the problem by stochastic control methods and the optimal portfolio can be explicitly characterized by stochastic integrals. The Monte Carlo simulations are implemented to compute the optimal portfolio allocations. The results show that state uncertainty have a great influence on optimal portfolio choice. The parameter uncertainty prompts the investor to hedge against unanticipated changes in the state variables.
\end{abstract}

\section{Keywords}

Portfolio choice; hidden regime switching; stochastic control methods; Monte Carlo simulation

\section{Introduction}

The traditional portfolio theory assumes that the asset returns are generated by a linear process with stable parameters. However, a large number of empirical researches have shown that returns often show a nonlinear and dynamic structural change. The financial market contains bull market and bear market. In different market states, the return and risk of assets are asymmetric ( Longin et al. ${ }^{[1]}$ and Ang et al. ${ }^{[2]}$ ).

Recently, many studies have turned to the optimal portfolio selection problem under Markovian regime-switching model. Empirically, the Markov regime switching model provide better fit to many economic and financial time series compared with their non-regimeswitching counterparts. Ang et al. ${ }^{[3-4]}$ and Liow et al. ${ }^{[5]}$ consider the international asset allocation problem under an observable Markov-modulated drift in a discrete-time setting. They show that the optimal investment weight depends on a variety of market states and investment horizon, and the regime-switching strategy dominated static strategies in an out-of-sample test. Guidolin et al. [6-7] promote the model of Ang et al. ${ }^{[3-4]}$ and assume regimes as unobservable. They find that optimal asset allocations vary considerably across these states and change over time as investors revise their estimates of the state probabilities. Guidolin ${ }^{[8]}$ consider international asset allocation under skew and kurtosis preferences with the presence of regimes in the return distribution. $\mathrm{Tu}{ }^{[9]}$ proposes a Bayesian framework for the decision problem under a regime-switching model. However, the studies mentioned above are discrete time models. Honda ${ }^{[10]}$ uses hidden Markov chain to describe the unobservable market state. He solves a 
continuous-time portfolio and consumption problem in which the expected return of a single risky asset depends on a two states hidden Markov chain. Optimal policies are computed using Monte Carlo methods. The optimal solution indicates that fluctuations in the estimated mean returns will cause hedging demand of assets. Furthermore, Liu [11] studies the optimal portfolio decisions in continuous time under ambiguity for regime switching mean returns for a long-horizon investor.

In this paper, we extend the model of Guidolin ${ }^{[6-7]}$ to study a continuous-time portfolio optimization problem in which the drift rate of the stock is two states hidden Markov modulated. An explicit solution is obtained from the stochastic dynamic programming principle. Our paper shares a similar set up but extends Honda's results in several directions by investigating explicit solution with stochastic control method, by entertaining economic implications of the model at the time focusing on the mathematical model, by performing Monte Carlo simulations to compute the optimal portfolios. We also solve the problem explicitly with all risk aversion coefficient, while Honda ${ }^{[10]}$ only showed the general solution to the case that the risk aversion coefficient is 0.5 .

\section{The Basic Model}

Let $(\Omega, F, P)$ be a probability space on which a standard Brownian motion $W_{t}$ and a two-state, continuous-time Markov chain $Y_{t}$ are defined. One risky and one riskless asset are available for investment. The return on riskless asset is a positive constant $r$. The risky asset price process $S_{t}$ satisfies

$$
d S_{t}=S_{t} \mu\left(Y_{t}\right) d t+S_{t} \sigma d W_{t}
$$

Where $\sigma$ is a constant. The expected return $\mu\left(Y_{t}\right)$ on the risky asset depends on a state variable $Y_{t}$.The state space is

$$
\{0,1\}
$$

while $\quad \mu_{H}=\mu(1), \mu_{L}=\mu(0)$

$\mu_{H}>\mu_{L}$, meaning that state 1 is the bull market with a high expected return and state 0 is the bear market with a low expected return. The infinitesimal generating matrix of $Y_{t}$ is $\left(\begin{array}{ll}-\lambda & \lambda \\ v & -v\end{array}\right)$, where $\lambda, v>0$.

Define $\pi_{t}=P\left(Y_{t}=1 \mid F_{t}^{s}\right)\left(\pi_{0}=x\right)$. $\pi_{t}$ is known as the filtered probability which is a prior probability that the current regime is the high expected return state. According to the definition of $Y_{t}$, we can see that $\pi_{t}=E\left[Y_{t} \mid F_{t}^{S}\right]$. So $\pi_{t}$ reflects the investor's expectations for the market state.

According to Theorem 9.1 of Liptser et al ${ }^{[12]}, \pi_{t}$ follows the stochastic differential equation

$$
d \pi_{t}=\left[\lambda-(\lambda+v) \pi_{t}\right] d t+\pi_{t}\left(1-\pi_{t}\right) \frac{\mu_{H}-\mu_{L}}{\sigma} d \hat{W}_{t}
$$

Where $\hat{W}_{t}$ is the standard Brownian motion with respect to $\left\{F_{t}^{S}\right\}$ defined by

$$
d \hat{W}_{t}=\frac{d S_{t}-S_{t} \hat{\mu}_{t} d t}{S_{t} \sigma}
$$

Where

$\hat{\mu}_{t}=E\left[\mu_{t} \mid F_{t}^{S}\right]=\pi_{t} \mu_{H}+\left(1-\pi_{t}\right) \mu_{L}$, then the process satisfies

$$
d \pi_{4}=\left[\lambda-(\lambda+v) \pi_{t}+\frac{\pi_{t}\left(\mu_{H}-\hat{\mu}\right)(\mu-\hat{\mu})}{\sigma^{2}}\right] d+\frac{\pi(\mu-\hat{\mu})}{\sigma} d W_{t}
$$

\section{Optimal portfolio choice model}

Time is continuous in the finite horizon $[\mathrm{t}$, 
T]. The utility function is the CRRA utility function. $\mathrm{Xt}$ is the wealth process, $\gamma$ is the risk aversion coefficient. A portfolio choice $b_{\mathrm{t}}$ is a $\left\{F_{t}^{S}\right\}$ adapted progressively measurable process such that $\int_{t}^{T}\left|b_{s}\right|^{2} d s<\infty \quad$.The budget constraint is given by

$$
d X_{t}=\left[r+b_{t}\left(\mu_{t}-r\right)\right] X_{t} d t+b_{t} \sigma X_{t} d W_{t}
$$

Which can be rewritten as

$$
d X_{t}=\left[r+b_{t}\left(\hat{\mu}_{t}-r\right)\right] X_{t} d t+b_{t} \sigma X_{t} d \hat{W}_{t}
$$

Based on the information set $\left\{F_{t}^{S}\right\}$, the investor makes portfolio decisions to maximize the expected lifetime utility

$$
\begin{aligned}
& \max _{b} E\left[U\left(X_{T}\right) \mid F_{t}^{S}\right] \\
& \text { s.t } d X_{t}=\left[r+b_{t}\left(\mu_{t}-r\right)\right] X_{t} d t+b_{t} \sigma X_{t} d W_{t}
\end{aligned}
$$

The optimal problem can be rewritten as follow

$$
\begin{aligned}
& \max _{b} E\left[U\left(X_{T}\right) \mid F_{t}^{S}\right] \\
& \text { s.t } d X_{t}=\left[r+b_{t}\left(\hat{\mu}_{t}-r\right)\right] X_{t} d t+b_{t} \sigma X_{t} d \hat{W}_{t} \\
& \qquad d \pi_{t}=\left[\lambda-(\lambda+v) \pi_{t}\right] d t+\pi_{t}\left(1-\pi_{t}\right) \frac{\mu_{H}-\mu_{L}}{\sigma} d \hat{W}_{t}
\end{aligned}
$$

Since $\hat{W}_{t}, \hat{\mu}_{t}, \pi_{t}$ are $\left\{F_{t}^{S}\right\}$ adapted, so we transform the incomplete information model into complete information model. Then, the standard methods can be used to solve the adjusted optimization problem

Define the value function $J^{(b)}(t, X, \Pi) @ \max _{b} E\left[U\left(X_{T}\right) \mid F_{t}^{S}\right]$.Fro $m$ the stochastic optimal control theory, the value function satisfies the following HJB partial differential equation

$$
\begin{aligned}
0=\max & \left\{J_{t}+\left[r+b_{t}(\hat{\mu}-r)\right] X_{t} J_{X}+\left[\lambda-(\lambda+v) \pi_{t}\right] J_{\pi}+\frac{1}{2} b_{t}^{2} \sigma^{2} .\right. \\
& +\frac{1}{2} \pi_{t}^{2}(1-\pi)^{2} \frac{\left(\mu_{H}-\mu_{t}\right)^{2}}{\sigma^{2}} J_{\pi t}+b_{t} \pi(1-\pi)\left(\mu_{H}-\mu_{L}\right.
\end{aligned}
$$

Thus, the optimal proportion of risky asset is

$$
b_{t}=\frac{\hat{\mu}_{t}-r}{\sigma^{2}}\left(\frac{-J_{X}}{J_{X X} X}\right)+\frac{\pi_{t}\left(1-\pi_{t}\right)\left(\mu_{H}-\mu_{L}\right)}{\sigma^{2}}\left(\frac{-J_{X \pi}}{J_{X X} X}\right)
$$

Under the power utility function, the optimal portfolio weight is

$$
b_{t}=\frac{\left(\hat{\mu}_{t}-r\right)}{\gamma \sigma^{2}}+\frac{\pi_{t}\left(1-\pi_{t}\right)\left(\mu_{\mathrm{H}}-\mu_{\mathrm{L}}\right) g_{\pi}\left(\pi_{t}, t\right)}{\sigma^{2} g\left(\pi_{t}, t\right)}
$$

Substitute $b_{t}$ into the HJB equation, then $g(\pi, t)$ satisfies the following partial differential equation

$$
\begin{aligned}
0= & \frac{1-\gamma}{\gamma}\left[r+\frac{1}{2 \gamma} \frac{\left(\hat{\mu}_{-}-r\right)^{2}}{\sigma^{2}}\right] g+\left[\lambda-(\lambda+\nu) \pi_{t}+\frac{1-\gamma}{\gamma} \pi_{t}\left(1-\pi_{t}\right)\left(\mu_{H}-\mu_{L}\right) \frac{\hat{\mu}_{-}-r}{\sigma^{2}}\right] g_{\pi} \\
& +g_{t}+\frac{\pi_{t}^{2}\left(1-\pi_{t}\right)^{2}\left(\mu_{H}-\mu_{L}\right)^{2}}{2 \sigma^{2}} g_{\pi}
\end{aligned}
$$

With $g(\pi, T)=1, \pi \in[0,1]$

The solution of Eq. (13) is given by the Feynman- Kac formula

$$
g(x, t)=E\left[\operatorname{ex}\left(\frac{1-\gamma}{\gamma} r(T-t)+\int_{t}^{T} \frac{1-\gamma}{2 \gamma^{2}} \frac{(\hat{\mu}(\pi)-r)^{2}}{\sigma^{2}} d s\right) \mid \pi=x\right]
$$

Where $\hat{\mu}\left(\pi_{t}\right)=\pi_{t} \mu_{H}+\left(1-\pi_{t}\right) \mu_{L}$.

Given that $\pi_{t}^{x}$ is the solution of Eq. (2) under the initial condition $\pi_{0}=x$. According to Protter ${ }^{[13]}, \pi_{t}^{x}$ is differentiable to $x$. Assuming that $B_{t}=\frac{\partial \pi_{t}^{x}}{\partial x}, g_{x}(x, t)$ can be expressed as follow based on Eq. (15)

$$
\begin{aligned}
g_{x}(x, t)= & E\left[\exp \left(\frac{1-\gamma}{\gamma} r(T-t)+\int_{t}^{T} \frac{1-\gamma}{2 \gamma^{2}} \frac{\left(\hat{\mu}\left(\pi_{s}\right)-r\right)^{2}}{\sigma^{2}} d s\right)\right. \\
& \left.\int_{t}^{T} \frac{1-\gamma}{\gamma^{2}} \frac{\left(\hat{\mu}\left(\pi_{s}\right)-r\right)\left(\mu_{H}-\mu_{L}\right)}{\sigma^{2}} B_{s} d s \mid \pi_{t}=x\right]
\end{aligned}
$$

$B_{t}$ satisfies the following stochastic partial differential equation with $\mathrm{B}_{0}=1$. 


$$
d B_{t}=-(\lambda+v) B_{t} d t+\left(1-2 \pi_{t}\right) \frac{\mu_{H}-\mu_{L}}{\sigma} B_{t} d W_{t}
$$

The optimal portfolio in (12) consists of two parts. The first term is the meanvariance portfolio, also known as myopic demand. The second component is the hedging portfolio against the future changes in the state dynamics of the mean return estimates. The hedging portfolio depends on $\sigma$ and $\mu_{\mathrm{H}}-\mu_{L} \quad, \quad \pi_{t}\left(1-\pi_{t}\right) \quad$, $g_{\pi}(\pi, t) / g(\pi, t)$. The hedging portfolio is large if $\mu_{\mathrm{H}}-\mu_{L}$ is large and $\sigma$ is small. Furthermore, if $\pi$ is near its boundaries and estimation risks are small, the hedging portfolio is small.

The size of the hedging portfolio is the product of $g_{\pi}(\pi, t) / g(\pi, t)$ and $\pi_{t}\left(1-\pi_{t}\right)\left(\mu_{H}-\mu_{L}\right) / \sigma^{2}$. Because of the boundary condition $g(\pi, T)=1$ for all $\pi$, for a short-horizon investor, $g_{\pi}$ is almost zero for all $\pi$, which makes the hedging portfolio is small. For longhorizon investors, $g_{\pi}(\pi, t) / g(\pi, t)$ can achieve a notable size.

\section{Numerical analysis}

We use Monte Carlo simulation to estimate $g$ and $g_{\pi}$. They are discretized as follows, Where $Z_{s}^{n}$ and $B_{s}^{n}$ are n-th sample path of $Z_{s}$ and $B_{s}$ respectively.

$$
\begin{aligned}
g(\pi, t)= & \frac{1}{N} \sum_{n=1}^{N} \exp \left(\frac{1-\gamma}{\gamma} r(T-t)+\int_{t}^{T} \frac{1-\gamma}{2 \gamma^{2}} \frac{\left(\hat{\mu}\left(\pi_{s}^{n}\right)-r\right)^{2}}{\sigma^{2}} d s\right) \\
g_{\pi}(\pi, t)= & \frac{1}{N} \sum_{n=1}^{N} \exp \left(\frac{1-\gamma}{\gamma} r(T-t)+\int_{t}^{T} \frac{1-\gamma}{2 \gamma^{2}} \frac{\left(\hat{\mu}\left(\pi_{s}^{n}\right)-r\right)^{2}}{\sigma^{2}} d s\right) \\
& \oint_{t}^{T} \frac{1-\gamma}{\gamma^{2}} \frac{\left(\hat{\mu}\left(\pi_{s}^{n}\right)-r\right)\left(\mu_{H}-\mu_{L}\right)}{\sigma^{2}} B_{s}^{n} d s
\end{aligned}
$$

In table 1, we show optimal portfolio choice under the different state probabilities and risk aversion coefficient for the parameters: $\mathrm{r}=0.05, \quad \mu_{1}=0.2$, $\mu_{2}=0.07, \lambda=v=0.25, \sigma=0.25, \mathrm{~N}=50000$. From part A, when $\gamma>1$, and $\pi_{0}$ increases gradually, investor will invest more wealth in the risky asset. When $\gamma<1$, the optimal portfolio weight increases and then decreases but re-increases with the rise of $\pi_{0}$. When $\pi_{0}=0.5$, the optimal portfolio weight decreases to the minimum.

From panel $\mathrm{B}$, at the time of $\pi_{0}$ closer to the boundary, the hedging portfolio decreases. The hedging portfolio position at the time of $\gamma=0.5$ is contrary to that at the time of $\gamma>1$. When $\gamma=0.5$ and $\pi_{0}>0.6$, the hedging portfolio is positive, and investor invests risk asset; when $\gamma=0.5$ and $\pi 0_{0}<0.6$, the hedging portfolio is negative, and investor short sells risk

\begin{tabular}{|c|c|c|c|c|}
\hline$\pi_{0}$ & $\gamma=0.5$ & $\gamma=1.5$ & $\gamma=2$ & $\gamma=4$ \\
\hline \multicolumn{5}{|c|}{ Panel A: optimal portfolio weight } \\
\hline 0 & 0.64 & 0.213 & 0.16 & 0.08 \\
\hline 0.3 & 1.147 & 0.773 & 0.633 & 0.358 \\
\hline 0.5 & 0.343 & 0.982 & 0.763 & 0.404 \\
\hline 0.8 & 4.321 & 1.285 & 0.949 & 0.464 \\
\hline 1 & 4.8 & 1.6 & 1.2 & 0.6 \\
\hline \multicolumn{5}{|c|}{ Panel B: hedging portfolio weight } \\
\hline 0 & 0 & 0 & 0 & 0 \\
\hline 0.3 & -0.915 & 0.143 & 0.161 & 0.122 \\
\hline 0.5 & -2.447 & 0.075 & 0.083 & 0.064 \\
\hline 0.8 & 0.353 & -0.038 & -0.042 & -0.032 \\
\hline 1 & 0 & 0 & 0 & 0 \\
\hline
\end{tabular}
asset. It is the other way around when $\gamma>1$.

Table 1 Optimal portfolio under various risk aversion coefficients

Table 2 shows the optimal portfolio and hedging portfolio under different investment horizons for the parameters $\gamma=1.5, \mathrm{r}=0.05, \mu_{\mathrm{H}}=0.2, \mu_{\mathrm{L}}=0.07, \lambda=\nu=0.25$, $\sigma=0.25$. If $\pi_{0}>0.5$ is considered as that the market is in the bull market and $\pi_{0}<=0.5$ is bear market, From panel A, the longer the investment horizon in the bear market, the optimal portfolio is larger. Because investor believes the market will pick up gradually although 
the current market is in the bear market, the longer the investment horizon, the greater the possibility that market picks up, and investor will increase the investment during the bear market. The situation in the bull market is just the opposite.

From panel $\mathrm{B}$, the longer the investment horizon, the greater the hedging portfolio, because the longer the investment horizon, the greater of the uncertainty of investment opportunity set, investor will pay more attention to this change.

Table 2 Optimal portfolio for various horizons

\begin{tabular}{|c|c|c|c|}
\hline$\pi_{0}$ & $\mathrm{~T}=1$ & $\mathrm{~T}=5$ & $\mathrm{~T}=10$ \\
\hline \multicolumn{4}{|c|}{ Panel A: optimal portfolio weight } \\
\hline 0 & 0.213 & 0.213 & 0.213 \\
\hline 0.3 & 0.621 & 0.664 & 0.773 \\
\hline 0.5 & 0.896 & 0.937 & 0.982 \\
\hline 0.8 & 1.315 & 1.331 & 1.285 \\
\hline 1 & 1.6 & 1.6 & 1.6 \\
\hline \multicolumn{4}{|c|}{ Panel B: hedging portfolio weight } \\
\hline 0 & 0 & 0 & 0 \\
\hline 0.3 & -0.008 & 0.035 & 0.143 \\
\hline \multicolumn{4}{|c|}{ Table 2 , cont } \\
\hline 0.5 & -0.010 & 0.029 & 0.075 \\
\hline 0.8 & -0.007 & 0.008 & -0.038 \\
\hline 1 & 0 & 0 & 0 \\
\hline
\end{tabular}

Table 3 shows optimal portfolio choice for different $\sigma$ and $\mu_{\mathrm{L}}$. The analysis on $\sigma$ is in the case of $\mathrm{T}=10, \gamma=1.5, \mathrm{r}=0.05$, $\mu_{\mathrm{H}}=0.2, \mu_{\mathrm{L}}=0.07$ and $\lambda=v=0.25$; analysis on $\mu_{\mathrm{L}}$ is in the case of $\mathrm{T}=10, \gamma=1.5$, $\mathrm{r}=0.05, \mu_{\mathrm{H}}=0.2, \lambda=\nu=0.25$ and $\sigma=0.25$.

As can be seen from the table, the smaller the $\sigma$, the more popular the risky assets if $\mu_{\mathrm{H}}$ and $\mu_{\mathrm{L}}$ are given. Thus, the smaller the $\sigma$, the greater the optimal proportion of risky assets. Panel B indicates that the hedging portfolio is more sensitive to $\pi_{0}$ when $\sigma$ is getting smaller, because investors are more sensitive to the market state when $\sigma$ decreases. From the table, we can see that the optimal portfolio is positively correlated with $\mu_{\mathrm{L}}$. The smaller the $\mu \mathrm{L}$, the investment proportion decreases and even the position is negative. Panel $\mathrm{B}$ indicates that absolute of the hedging portfolio is greater than those in other two cases when the expected return is negative in the worst case and $\mu_{\mathrm{L}}=-0.07$.

Table 3 Optimal portfolio for various $\sigma$ and $\mu_{\mathrm{L}}$

\begin{tabular}{lll|ll}
\multicolumn{5}{l}{ Panel A: optimal portfolio weight } \\
\hline$\pi_{0}$ & $\sigma=0.15$ & $\sigma=0.25$ & $\mu_{\mathrm{L}}=-0.07$ & $\mu_{\mathrm{L}}=0.07$ \\
\hline 0 & 0.593 & 0.213 & -1.28 & 0.213 \\
0.3 & 2.613 & 0.773 & -0.092 & 0.773 \\
0.5 & 2.870 & 0.982 & 0.179 & 0.981 \\
0.8 & 3.471 & 1.285 & 0.457 & 1.285 \\
1 & 4.444 & 1.6 & 1.6 & 1.6 \\
\hline \multicolumn{5}{l}{ Panel B: hedging portfolio weight } \\
\hline$\pi_{0}$ & $\sigma=0.15$ & $\sigma=0.25$ & $\mu_{\mathrm{L}}=-0.07$ & $\mu_{\mathrm{L}}=0.07$ \\
\hline 0 & 0 & 0 & 0 \\
0.3 & 0.865 & 0.143 & 0.324 & 0.143 \\
0.5 & 0.352 & 0.075 & 0.019 & 0.075 \\
0.8 & -0.203 & -0.038 & -0.566 & -0.038 \\
1 & 0 & 0 & 0 & 0 \\
\hline
\end{tabular}

\section{Conclusion}

In this paper, we analyze optimal portfolio choice extends the analysis in a continuous-time setting, where the unobservable mean return of a risky asset follows a two-state continuous-time Markov chain. By the dynamic programming and Feynman-Kac formula, the optimal portfolio can be characterized by the expectations of stochastic integrals. The Monte Carlo simulations are implemented to compute the optimal portfolio allocations. The results show that, state uncertainty and dynamic learning have a great influence on optimal portfolio choice. The hedging portfolio of a long-time-horizon investor is larger than that of a short-time-horizon investor. The volatility of risk asset is smaller; the slight change of $\pi_{0}$ will cause a larger change of hedging portfolio. The optimal portfolio allocation can decrease or vary non-monotonically in the investment horizon, which casts in doubt the conventional wisdom that young investor should allocate more wealth into equity. In addition, the optimal portfolio is less sensitive to the change in the state variable for a risk-averse investor. 


\section{References}

[1] Longin F, Solnik B. Extreme correlation of international equity markets [J].The Journal of Finance, 2001, 56 (2):649-676.

[2] Ang A, Chen J. Asymmetric correlations of equity portfolios [J]. Journal of Financial Economics, 2002, 63 (3):443-494.

[3] Ang A, Bekaert G. International asset allocation with regime shifts [J]. Review of Financial Studies, 2002, 15 (4):1137-1187.

[4] Ang A, Bekaert G. How regimes affect asset allocation [J]. Financial Analysts Journal, 2004, 60 (2):8699.

[5] Liow K, Zhu H. Regime switching and asset allocation: evidence from international real estate security markets [J]. Journal of Property Investment \& Finance, 2007, 25 (3): 274-288.

[6] Guidolin M, Timmermann A. Economic implications of bull and bear regimes in UK stock and bond returns [J]. Economic Journal, 2005,115 (500):111-143.

[7] Guidolin M, Timmermann A. Asset allocation under multivariate regime switching [J]. Journal of Economic Dynamics \& Control, 2007, 31 (11):3503-3544.

[8] Guidolin M, Timmermann A. International asset allocation under regime switching, skew, and kurtosis preferences [J]. Review of Financial Studies, 2008, 21 (2):889935.

[9] Tu J. Are bulls and bear markets economically important? [J]. Management Science, 2010, 56 (7): 1198-1215.

[10] Honda T. Optimal portfolio choice for unobservable and regimeswitching mean returns [J]. Journal of Economic Dynamics and Control,
2003, 28(1): 45-78.

[11] Liu H. Dynamic portfolio choice under ambiguity and regime switching mean returns [J]. Journal of Economic Dynamics and Control, 2011, 35(4): 623-640.

[12] Liptser R S, Shiriaev A N, Shiryaev A N. Statistics of Random Processes II: II. Applications [M].Springer, 2001.

[13] Protter P. Stochastic integration and differential equations $[\mathrm{M}]$. Springer, 1990. 